\title{
Antibacterial Silver Embedded Nanofibers for Water Disinfection
}

\author{
Thanaa Shalaby ${ }^{1}$, Ola Mahmoud ${ }^{2}$, Affaf Al-Oufy ${ }^{3}$ \\ ${ }^{1}$ Medical Biophysics Dept., Medical Research Institute, Alexandria University, Alexandria, Egypt \\ ${ }^{2}$ Microbiology Dept., Medical Research Institute, Alexandria University, Alexandria, Egypt \\ ${ }^{3}$ Textile Eng. Dept., Faculty of Engineering, Alexandria University, Alexandria, Egypt \\ Email address: \\ th_shalaby@yahoo.com (T. Shalaby),olakader@hotmail.com (O. Mahmoud), affafaloufy@yahoo.com (A. A-Oufy)
}

\section{To cite this article:}

Thanaa Shalaby, Ola Mahmoud, Affaf Al-Oufy. Antibacterial Silver Embedded Nanofibers for Water Disinfection. International Journal of Materials Science and Applications. Vol. 4, No. 5, 2015, pp. 293-298. doi: 10.11648/j.ijmsa.20150405.13

\begin{abstract}
Anti-microbial nanofibers can be synthesized by incorporating nanobiocides such as silver nanoparticles into nanofibers. Bacterial contamination of water is a serious public health concern because it causes numerous diseases and some aesthetic problems such as malodor in water. Durable antibacterial Ag/polyacrylonitrile (Ag/PAN) hybrid nanofibers were prepared by electrospinning. SEM and EDX spectra were used to study the morphology and surface elemental composition of the nanofibers. The Ag/PAN nanofibers exhibited slow and long lasting silver ion release. The resultants polymers were then tested for their ability to destroy bacteria in water; antimicrobial tests indicated that the prepared nanofibers have a high bactericidal effect. Accordingly, these results highlight the potential use of these nanofibrous mats as antimicrobial agents.
\end{abstract}

Keywords: Polyacrylonitrile, Electrospinning, Nanofibers, Nanosilver, Antimicrobial, Water Disinfection

\section{Introduction}

The development of energy-efficient water treatment technology has become an important area of research as it offers a solution to the increasingly limited water supplies available to the world's growing population and industry. Nanotechnology is the discipline of manipulating matter at the nanoscale (1-100 $\mathrm{nm})$, yielding nanoparticles or materials that often possess novel biological, physical or chemical properties[1] water treatment[2 and 3].

Nanofibers have excellent filtration properties, and due to the variety of polymers that can be used to fabricate nanofibers, and the versatility of being able to add functional molecules and chemical groups to the nanofibers, make nanofibers applicable to sanitation and purification of water. Anti-microbial nanofibers can be synthesized by incorporating nanobiocides such as silver nanoparticles into the nanofibers. The synthesis of nanofibers containing metal nanoparticles is well researched because of the advantages involved with combining the functional properties of metal nanoparticles with the widely applicable properties of nanofibers [4].

For generation of nano-Ag-bearing nanofibres, silver can be added to the spinning solution either in form of nanoparticles [5] or as silver-ions which can be reduced to nano-Ag by a corresponding post-treatment [6 and 7]. Reduction of ionic silver can be achieved i) by subsequent UV-irradiation of the fiber webs [8], ii) by the use of reducing agents as hydrazinium hydroxide [8] and hydrogen [9] as well as iii) by heat-treatment of the nanofibres [10].

In the past few years, silver-containing electrospun nanofibers have attracted interest as a novel form of antimicrobial material [11 and 12]. Electrospinning is a wellestablished process for fabrication of nanofiber mats with high surface areas, large volume-to mass ratios, and high porosity [13]. This process has been demonstrated to be suitable for manufacturing scale-up for low-cost mass production [14]. Silver is a widely-used and recognized broad spectrum biocidal agent that is effective against bacteria, fungi and viruses but is non-toxic to human cells [15 and 16]. The combination of the high specific surface area and fineness of electrospun nanofibers with the biocidal activity of Ag nanoparticles results in a superior and versatile antimicrobial material [17 and 18].

The aim of this study is to prepare antimicrobial PAN nanofibers containing silver nanoparticles (Ag-NPs) by simple electrospinning technique and demonstrating their antibacterial properties for use in water disinfection Escherichia coli (E. coli) was chosen in this study as 
indicators of fecal contamination.

\section{Materials and Methods}

\subsection{Materials}

Polyacrylonitrile (PAN, Mw 900,000) and N,Ndimethylformamide (DMF) and Silver nitrate $\mathrm{AgNO}_{3}$ were obtained from Sigma-Aldrich (St. Louis, MO, USA) and used as received.

Pure slant cultures Escherichia coli (E. Coli) were obtained from the Microbiology Department, Medical Research Institute (MRI) Alexandria, Egypt. E. coli ATCC 25922 was selected as indicators of fecal contamination of water. Nutrient broth was used as the growing medium.

\subsection{Preparation and Characterization of Plain and Silver- Embedded Nanofibers}

A polymer solution of $7 \%(\mathrm{wt} / \mathrm{wt})$ PAN in DMF was prepared. Dimethylformamide was heated up to $50^{\circ} \mathrm{C}$ and stirred while PAN was added gradually. The mixture was stirred at $50^{\circ} \mathrm{C}$ for $\sim 2$ hours until a clear, slightly yellow solution was obtained. Finally, 5\% (wt $/ \mathrm{v}) \mathrm{AgNO}_{3}$ was thoroughly mixed into the polymer solution. DMF assists in the reduction of $\mathrm{AgNO}_{3}$ silver ions to silver nanoparticles. The solution was immediately collected into a $10 \mathrm{ml}$ syringe equipped with a 24 stainless steel blunted tip needle. PAN nanofibers were prepared using the electrospinning technique in an air-conditioned laboratory. The process conditions were kept at an ambient temperature of $22^{\circ} \mathrm{C}$ and relative humidity of $<65 \%$. The syringe was fixed on an electric syringe pump set to maintain a constant feed rate of $1.5 \mathrm{ml} / \mathrm{h}$. A highvoltage power supply (Gamma high voltage, Inc., USA) was employed to apply positive charge to the needle, and a grounded metal plate covered with aluminum foil served as the collector. The voltage used for electrospinning was $22 \mathrm{kV}$. The distance between the needle tip and collector was $15 \mathrm{~cm}$.

PAN nanofibers containing $\mathrm{AgNO}_{3}$ and already reduced silver nanoparticles were collected on the plate. Subsequently, the nanofibers were exposed to UV irradiation for one hour to reduce any remaining silver ions in the nanofibers to silver nanoparticles. Plain PAN nanofibrous mat without silver nanoparticles were used as a negative control in the antimicrobial tests. The PAN nanofibers with and without $\mathrm{Ag}$ NPs were characterized using scanning electron microscope (SEM, Joel, JSM-6360 LA-Japan), the presence of Ag NPs attached with PAN nanofibers was detected using energy dispersive $\mathrm{x}$-ray spectroscopy (EDX).

\subsection{Silver Ion Release}

Silver ion release behavior of the $\mathrm{Ag} / \mathrm{PAN}$ hybrid nanofibers was determined by atomic absorption spectrometry (AAS). A small piece of electrospun nanofibous mat (approximately $100 \mathrm{mg}$ ) was placed in a glass container, and $150 \mathrm{ml}$ deionized water was added into the container as the release medium. The container was sealed and agitated to insure complete immersion of the nanofibrous mat, and then incubated at $37^{\circ} \mathrm{C}$. The deionized water was collected every $24 \mathrm{hrs}$, and silver ion concentration in the solution was measured using a PerkinElmer AA300 AA spectrometer (Thermo Scientific ICE $3500)$.

\subsection{Microbiological Study}

Bacteria were grown aerobically in nutrient broth at $37^{\circ} \mathrm{C}$ for $12 \mathrm{~h}$ [19]. The cultures were centrifuged and the cells were washed and suspended in distilled water, reaching a final concentration of $10^{5}-10^{6} \mathrm{CFU} / \mathrm{mL}$. The antimicrobial susceptibility of PAN nanofibers with Ag NPs was evaluated using the disc diffusion method and a contact test.

\subsubsection{The Disc Diffusion Method}

Müller-Hinton Agar was prepared from a commercially available dehydrated medium $\left(\right.$ Oxoid $\left.^{\circledR}\right)$ according to manufacturer's instructions. The dried surface of a MuellerHinton agar plate was inoculated with E. coli by swabbing over the entire sterile agar surface. Two sterilized samples of PAN nanofiber (one containing Ag nanoparticles and one without $\mathrm{Ag}$ nanoparticles, used as control) were cut into small squares and placed on the surface of the inoculated media. The plates were incubated at $37^{\circ} \mathrm{C}$ for 24 hours. Following incubation, plates were examined in order to identify zones of no growth (halos around the fragments) characteristic for antimicrobial activity.

\subsubsection{Contact Test}

The antimicrobial activity of Ag-Nps loaded nanofibers was tested by immobilizing the nanofibers onto filters of a Millipore filtration (dead-end) cell (Nalge Nunc International, USA). A test water sample was prepared by inoculating $10^{6}$ cells $/ \mathrm{ml}$ E. coli into $250 \mathrm{ml}$ of sterile distilled $\mathrm{H}_{2} \mathrm{O}$. Water samples were then filtered through $0.22 \mu \mathrm{m}$ filters, with either plain PAN nanofibers, or PAN nanofibers containing silver nanoparticles immobilized on the filter surface, a rubber ring was used to support the membrane edge. The filter pore size of $0.22 \mu \mathrm{m}$ excluded bacterial cells, causing the cells to remain in contact with the nanofibers. After filtration, the viability of the cells remaining on the nanofibers with silver nanoparticles was investigated, by determining the viable cells recovered from the nanofibers. This was done by washing of nanofibers after contact times of 3, 6, 12 and 24 hours. Washed bacterial samples were plated onto solid nutrient agar plates then incubated overnight at $37^{\circ} \mathrm{C}, \mathrm{CFU}$ was visualized.

\section{Results and Discussion}

\subsection{Morphological Study (SEM)}

The color of PAN/AgNo 3 nanofibrous web changed from white to yellow, when irradiated with UV irradiation for $1 \mathrm{hr}$, as illustrated in "Fig. 1". The change in color of PAN polymer nanofibers is a result of the synthesized Ag NPs on the polymeric nanofiber. Ag NPs have an interesting feature, so called surface plasmon resonance (SPR) phenomenon, 
which occurs when light is reflected off thin metal film or NPs.

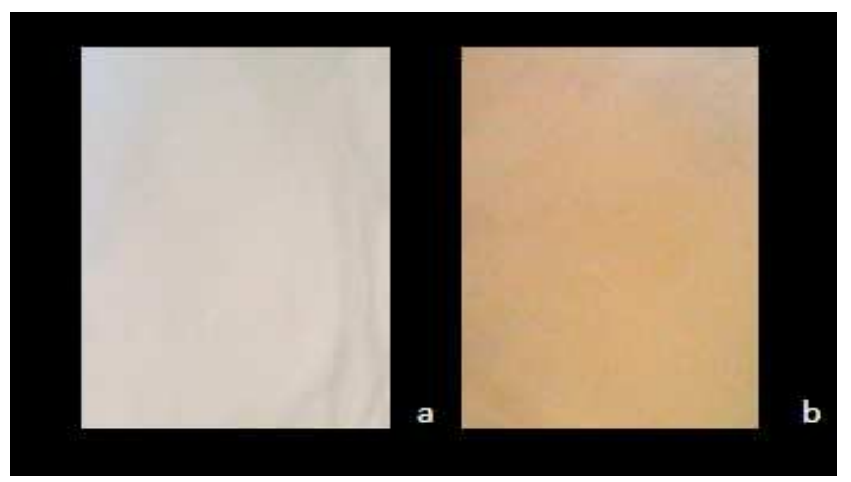

Figure 1. Photographs of PAN nanofibrous web (a) without Ag NPS and (b) embedded with Ag NPs.

SEM images of PAN nanofibers with and without Ag NPs are shown in "Fig. 2 and 3". As illustrated in Fig. 2, the PAN nanofibers of $7 \%$ gave a smooth, free beads structures and homogenous morphology. The calculated average diameter of the used nanofibers was around $400 \mathrm{~nm}$.

The PAN nanofibers maintained its morphology upon loading with silver nanoparticles. "Fig. 3-a" shows a highmagnification SEM image of PAN nanofibers containing Ag

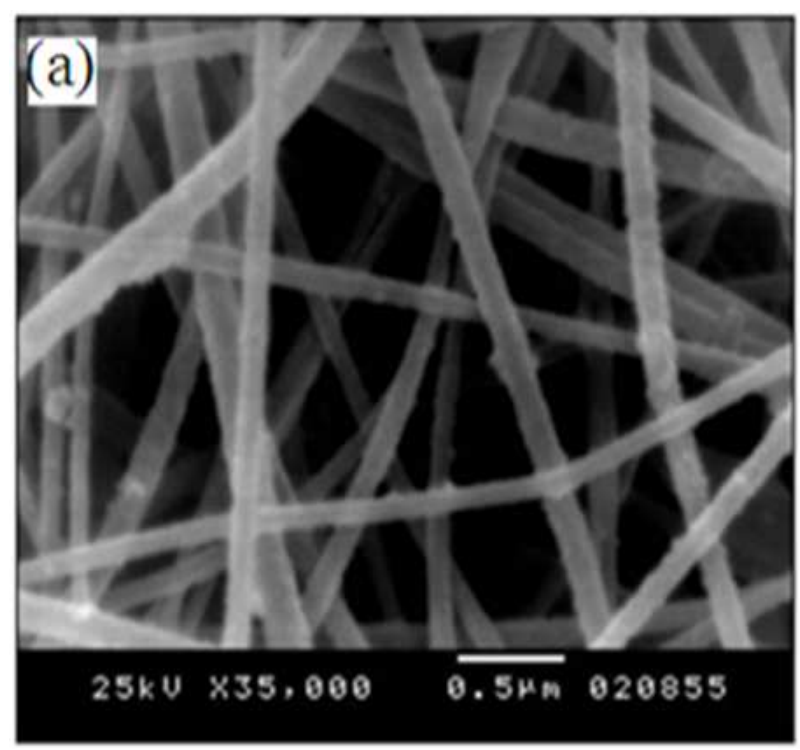

Figure 3. SEM/EDX of Ag NPs loaded PAN nanofibers ( $a$ and $b$ ).

\subsection{Silver Ion Release}

For silver-based antibacterial properties, the most critical factor is the silver release behavior, which can inhibit the growth of bacteria. It has been reported that a steady and prolonged release of silver at a concentration level as low as 0.1 part per billion (ppb) can render effective antimicrobial activity [20]. In this research work, silver release rates of $\mathrm{Ag} / \mathrm{PAN}$ nanofibers were investigated in deionized water. In an aqueous environment, the $\mathrm{Ag}$ nanoparticles embedded in the nanofibers are released into the solution in form of silver ions. "Fig. 4" demonstrates the silver release profiles of nanoparticles, in which the Ag nanoparticles can be observed on the surface of the nanofibers. "Fig. 3-b" shows the EDX spectra of these nanofibers. The EDX analysis of PAN nanofibers with silver nanoparticles shows a distinctive energy peak at around $3 \mathrm{KeV}$, characteristic of silver. The peak for elemental $\mathrm{Ag}$ is additional evidence for the formation and presence of $\mathrm{Ag}$ nanoparticles.

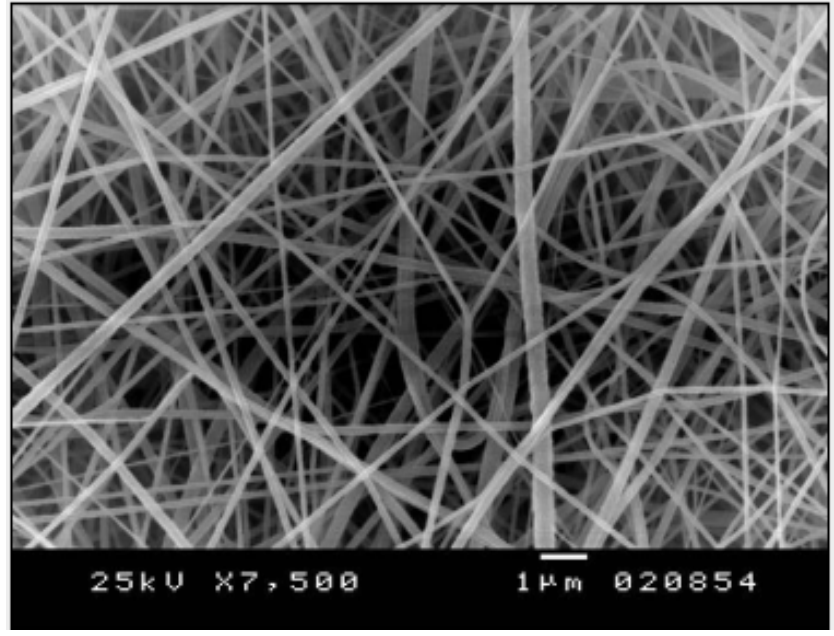

Figure 2. SEM of PAN nanofibers with concentration $7 \%(w t \%)$ in DMF as a solvent.

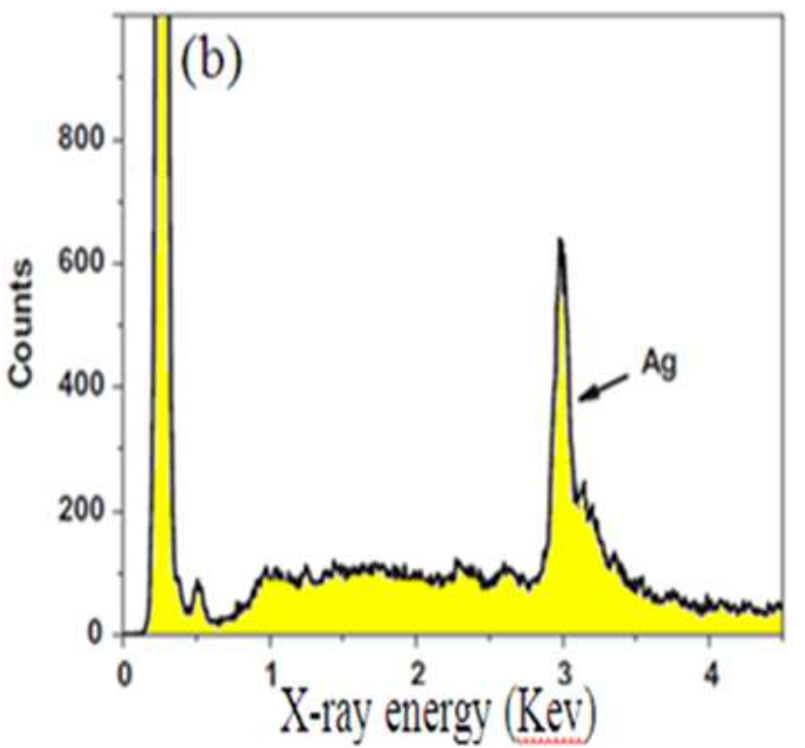

Ag/PAN hybrid nanofibers over 9 days. The release rate (i.e., the slope of the curve $\alpha$ ) is relatively high $\left(\alpha_{1}\right)$ in the first day and then decreases $\left(\alpha_{2}\right)$ as the $\operatorname{Ag}$ nanoparticles on the surface of nanofibers are readily available to react with water. After the initial release of surface silver, the release process transitions to a diffusion-based release from nanoparticles embedded inside the nanofiber matrix. The silver release rate and cumulative release amount indicate that Ag/PAN nanofibers prepared by electrospinning can release sufficient silver to exhibit sustained antibacterial activity. Therefore, one can recommend these nanofibers for long term contact 
with water operations; like antimicrobial water filters.

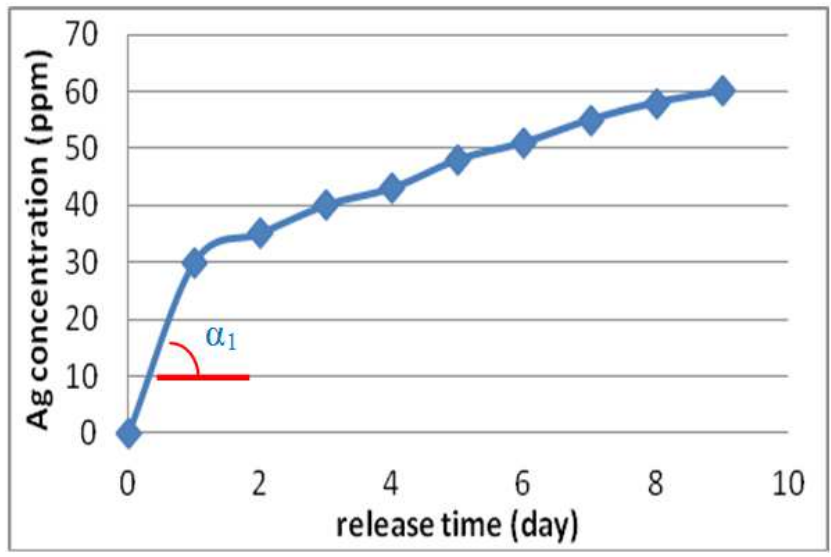

Figure 4. Silver release profile of Ag/PAN hybrid nanofibers over 9 days.

\subsection{Microbiological Study}

As far as antimicrobial agents are considered, silver is considered a material of choice, and its forms are potent antimicrobials against various bacterial species. The exact mechanism of how silver participates in distortion of bacteria is unfortunately still unclear. Silver nanoparticles adhere to the microbial cell membrane where it interacts with sulfur-containing proteins. Nanoparticles can also penetrate the cell membrane, where it interacts with phosphorous-containing DNA and attack thiol groups of respiratory chain enzymes, inhibiting cell division and respiration, finally leading to cell death [21 and 22]. However, generally it is believed that sliver mainly makes denaturation and oxidation for cell organelles which lead to suppress the cell division. It is well known fact when a critical amount of antibacterial compound (capable of inhibiting microbial growth) is in contact with bacterial strains the clear area near that contact is formed and these areas are referred as zones of inhibition [23].

"Fig. 5" shows the incubated petri plates of E. coli grown in presence of square nanofiber patches after $24 \mathrm{hrs}$. From that Figure, it is clearly observed the presence of clear zones around the square nanofiber patches containing $\mathrm{Ag}$ NPs while as in case of plain PAN nanofiber these zones were missing. Formation of clear zones around the nanofiber patches is an indicator for inhibition of bacterial colonies near the vicinity of nanofiber patch edges. Basically, due to presence of silver in the nanofiber patch, it may lead to kill the microorganism around the patch which results in formation of clear circular zones.

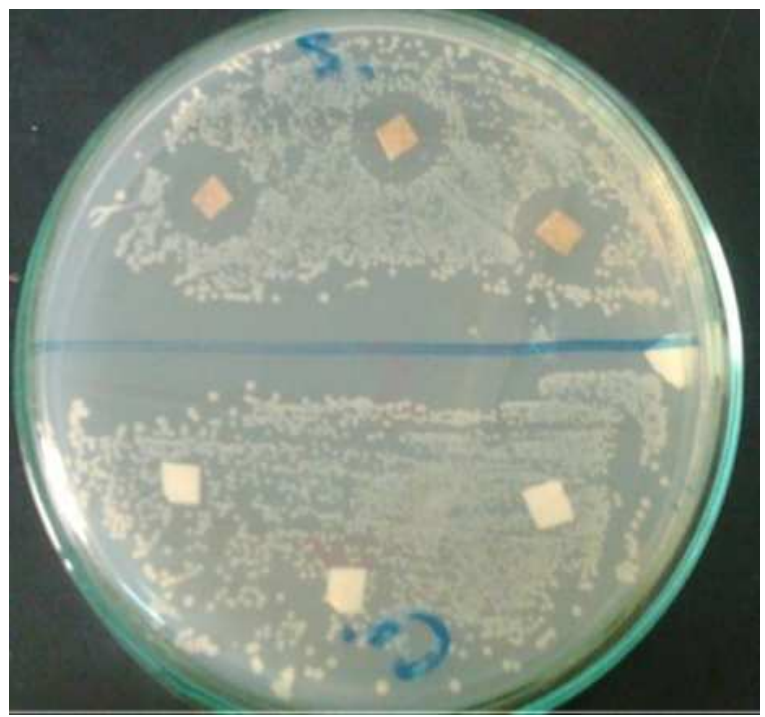

Figure 5. Antimicrobial activities of PAN nanofibers against Escherichia coli. Upper part illustrates the Ag NPs embedded PAN nanofibers, lower part illustrates plain PAN nanofibers. Zone of inhibition is visible around PAN disks containing Ag NPs.

Antimicrobial activity of the PAN nanofibers loaded with silver nanoparticles was also proven by contact tests. The tests illustrated excellent antimicrobial activity of the Agbearing nanofibres. "Fig. 6" shows the inhibition of bacterial growth after different contact periods $(3,6,12$ and 24 hours) with nanofibes incorporated with Ag NPs; it is clear that, as the contact time increased the bacterial colonies decreased. "Fig. 7" shows that, PAN nanofibres incorporated with nano-Ag inhibited the growth of E.coli in opposition to the references PAN nanofibers, after a contact time of 18 hours with the PAN nanofibers loaded with silver nanoparticles, the numbers of colonies of E. coli were significantly reduced $(>98 \%)$ and reach $100 \%$ after contact time of 24 hours.
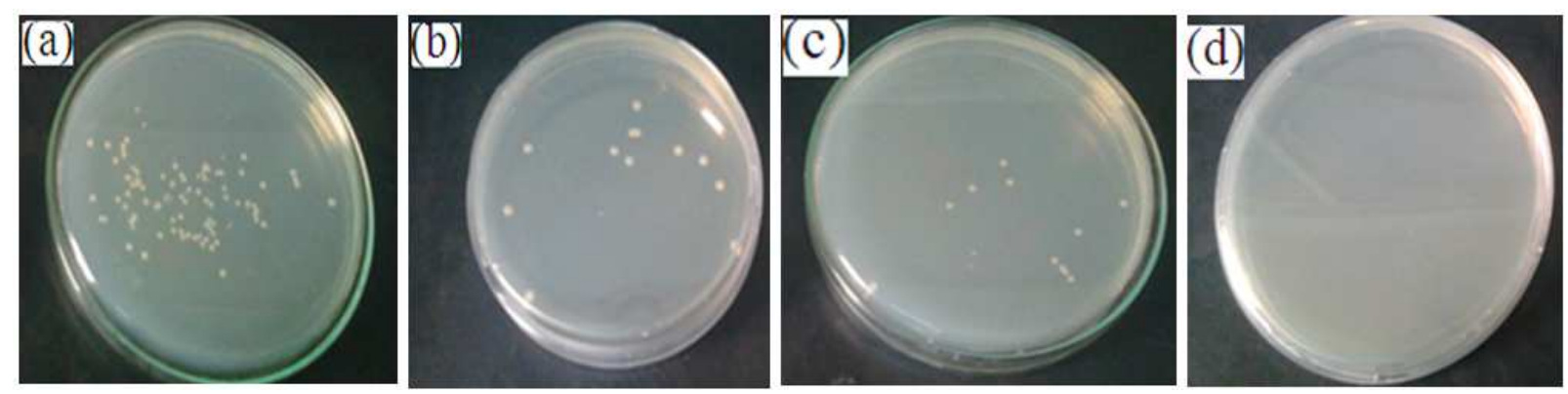

Figure 6. Growth inhibition of E. coli grown on nutrient agar plate as a function of contact time with PAN nanofibers loaded with Ag NPs for 3 hrs (a), 6 hr (b), $12 \mathrm{hr}$ (c) and $24 \mathrm{hr}$ (d) before the incubation period. 


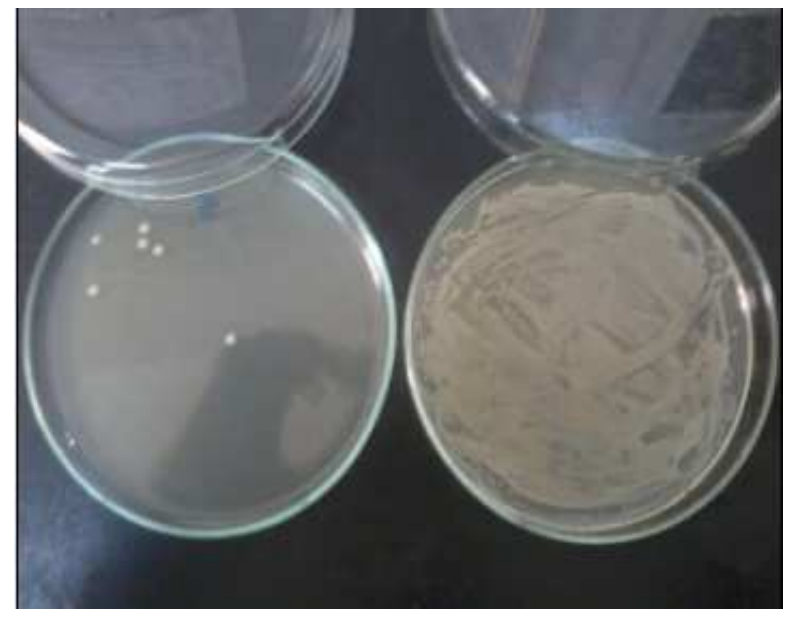

Figure 7. Contact test result for E. coli after 24 hrs incubation. The right plate represents the negative control plate. The left plate represents the count after contact with PAN loaded with Ag NPs for 18 hours.

\section{Conclusions}

The potential of incorporating antimicrobial activity in the nanofilters would be explored to facilitate destruction of pathogenic microorganisms. Our research work proved that, PAN nanofibers are excellent candidate for carrying silver nanoparticles and for being used in water treatment. PAN nanofibers; embedded with silver nanoparticles, were successfully fabricated by electrospinning, and that was confirmed by SEM analysis. PAN nanofibers containing silver nanoparticles had excellent antimicrobial activity, a high bacterial removal was observed with Ag-NPs loaded PAN nanofibers. The experiments suggest the possibility of using such material in drinking water purification, air filtration, domestic and industrial air quality management, antibacterial packaging, etc. The technology can be implemented with minimum infrastructure and therefore offers a solution to the affected people without intermediaries. Along with other nanoparticles, the technology can offer complete antimicrobial solutions for rural communities.

\section{Acknowledgments}

This work is part of a research project funded by the Research Enhancement Program (Alex Rep) of Alexandria University, Alexandria, Egypt. Project Code: ENV-10.

\section{References}

[1] Theron J., Walker JA., and Cloet TE. (2008) "Nanotechnology and water treatment applications and emerging opportunities". Crit. Rev. Microbiol. 34, 43-69

[2] Savage N. and Diallo M. S. (2005). Nanomaterials and water purification: Opportunities and challenges. J Nanopart Res 7, 331-342.
[3] Bottero J., Rose J., Wiesner M. R. (2006) Nanotechnologies: Tools for sustainability in a new wave of water treatment processes. Integrated Environ Assess Manag 2, 391-395.

[4] Zhang LF, Luo JE, Menkhaus TJ, Varadaraju H, Sun YY, Fong H. Antimicrobial nano-fibrous membranes developed from electrospun polyacrylonitrile nanofibers. J Memb Sci 2011;369: 499-505.

[5] Park S, Bae H, Xing Z, Kwon O, Huh M, Kang I. (2009) Preparation and properties of silver-containing nylon 6 nanofibers formed by electrospinning. J Appl Polym Sci 2009;112(4):2320-6.

[6] Jeon H, Kim J, Kim T, Kim J, Yu W, Youk J. (2008) Preparation of poly(ecaprolactone)- based polyurethane nanofibers containing silver nanoparticles. Appl Surf Sci; 254(18):5886-90.

[7] Kumar A, Chhatra R, Pandey P. (2010) Synthesis of click bile acid polymers and their application in stabilization of silver nanoparticles showing iodide sensing property. Org Lett; 12(1):24-7.

[8] Luong N, Lee Y, Nam J. (2008) Highly-loaded silver nanoparticles in ultrafine cellulose acetate nanofibrillar aerogel. Eur Polym J; 44(10):3116-21.

[9] Li S, Shen Y, Xie A, Yu X, Qiu L, Zhang L, et al. (2007) Green synthesis of silver nanoparticles using Capsicum annuum L. extract. Green Chem; 9(8):852-8.

[10] Sharma V, Yngard R, Lin Y. (2009) Silver nanoparticles: green synthesis and their antimicrobial activities. Adv Colloid Interface; 145(1-2):83-96.

[11] Xu X, Yang Q, Wang Y, Yu H, Chen X, Jing X. (2006) Biodegradable electrospun poly(L-lactide) fibers containing antibacterial silver nanoparticles. Eur Polym J; 42(9):2081-7.

[12] Kim J, Kuk E, Yu K, Kim J, Park S, Lee H, et al. (2007) Antimicrobial effects of silver nanoparticles. Nanomed Nanotechnol; 3(1):95-101.

[13] Reneker D, Yarin A. (2008) Electrospinning jets and polymer nanofibers. Polymer; 49(10):2387-425.

[14] Greiner A, Wendorff J. (2007) Electrospinning: a fascinating method for the preparation of ultrathin fibers. Angew Chem Int Ed; 46(30):5670-703.

[15] Rai M, Yadav A, Gade A. (2009) Silver nanoparticles as a new generation of antimicrobials. Biotechnol Adv; 27(1):76-83.

[16] Feng Q. L., Wu J., Chen G. Q., Cui F. Z., Kim T. N., Kim J. O. (2000) A mechanistic study of the antibacterial effect of silver ions on Escherichia coli and Staphylococcus aureus. J Biomed Mat Res 52, 662-668.

[17] Pim-on R, Nuttaporn P, Pitt S. (2010) Preparation, characterization, and antibacterial properties of electrospun polyacrylonitrile fibrous membranes containing silver nanoparticles. J Appl Polym Sci; 116(4):1967-76.

[18] Castellano J, Shafii S, Ko F, Donate G, Wright T, Mannari R, et al (2007) Comparative evaluation of silver-containing antimicrobial dressings and drugs. Int Wound; 4(2):114-22.

[19] Pal S, Tak YK, Song JM. (2007). Does the antibacterial activity of silver nanoparticles depend on the shape of the nanoparticle? A study of the Gram negative bacterium Escherichia coli. Applied and Environmental Microbiology; vol 73, No 6, 1712-1720. 
[20] Kumar R, Munstedt H. (2005) Silver ion release from antimicrobial polyamide/silver composites. Biomaterials; 26(14):2081-8

[21] De Santa Maria L, Santos A, Oliveira P, Barud H, Messaddeq Y, Ribeiro S.(2009) Synthesis and characterization of silver nanoparticles impregnated into bacterial cellulose. Mater Lett; 63(9-10):797-9.
[22] Chen JP, Chiang Y. Bioactive electrospun silver nanoparticlescontaining polyurethane nanofibers as wound dressings. $\mathrm{J}$ Nanosci Nanotechno 2010; 10:7560- 4.

[23] Sheikh FA. (2009) Electrospun antimicrobial polyurethane nanofiber containing silver nanoparticles for biotechnological applications., Macromolecular Research, vol 17, No 9, pp 688-696. 